\title{
El reto de la formación en competencias digitales: el Proyecto SOLA y su papel en la UDIMA
}

\section{Extracto:}

\section{Sumario}

1. Introducción

2. La internacionalización de la UDIMA, contexto para el Proyecto SOLA

3. El Proyecto SOLA

4. El caso de la UDIMA en el Proyecto SOLA

5. Conclusiones

6. Bibliografía
El proyecto SOLA (cuya sigla significa simple open learning advancement) es un Proyecto del denominado Programa Erasmus+ (KA2-Cooperation and Innovation for Good Practices), convocado por la Comisión Europea. Su finalidad es, principalmente, perfeccionar las competencias digitales y de las tecnologías de la información y la comunicación (TIC) del profesorado. Además, tiene como otros objetivos ayudar en la disposición y en el desarrollo de recursos abiertos educativos, conectar aulas e implementarles dispositivos y contenidos digitales, y, por último, fomentar el uso de las TIC en instituciones educativas mediante la movilización de profesores, estudiantes y demás participantes sociales, socios económicos y familia. La UDIMA participa en el Proyecto SOLA junto a otras instituciones formativas europeas de Alemania, Austria, Italia, Portugal, Países Bajos, Polonia y República Checa, que han diseñado la estructura y los contenidos de varios cursos formativos en la modalidad semipresencial o blended learning, centrados principalmente sobre las dimensiones más importantes de sostenibilidad del desarrollo turístico, medioambiental, económico y sociocultural.

Palabras clave: Proyecto SOLA (simple open learning advancement), blended learning, tecnologías de la información y la comunicación (TIC), e-learning, turismo sostenible.
Fecha de entrada: 28-06-2016

Fecha de aceptación: 15-11-2016 


\section{The challenge of training in digital competences: the SOLA} Project and its paper in

\section{the UDIMA}

\section{Abstract:}

SOLA Project (simple open learning advancement) is a project organized by the European Commission and then accepted into the Erasmus+ (KA2-Cooperation and Innovation for Good Practices) program. Its purpose is mainly to perfect digital skills and information and communication technologies (ICT) teacher, also has other objectives assist in the provision and development of open educational resources, connect classrooms and to install and implement them devices and digital content, and finally encourage the use of ICT in education institutions by mobilizing teachers, students and other participants social, economic and family members. UDIMA participates in the SOLA Project with other European training institutions such as Germany, Austria, Italy, Portugal, the Netherlands, Poland and the Czech Republic and have designed the structure and content of several training courses in a blended format or blended learning, mainly established on key dimensions of sustainable tourism, environmental, economic and socio-cultural development.

Keywords: SOLA Project (simple open learning advancement), blended learning, information and communication technologies (ICT), e-learning, sostenible tourism.

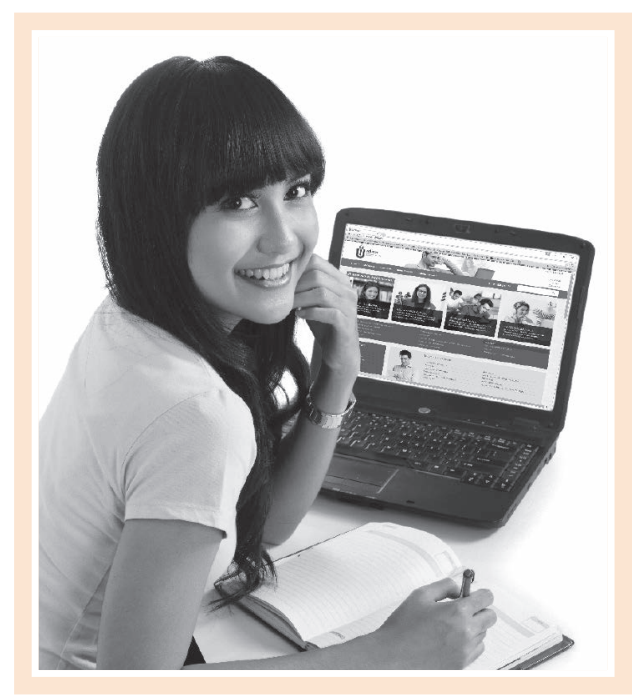

\section{INTRODUCCIÓN}

La institución coordinadora del proyecto es el Berufsförderungsinstitut (BFI). Sin ánimo de extendernos en la descripción de este organismo (puesto que dicha información está contenida en su página web)², podemos decir brevemente que se trata de una entidad privada situada en Austria, cuyo principal objetivo pivota sobre la innovación en los métodos de la enseñanza permanente mediante la utilización intensiva de la tecnología. Así, se configura como una institución que proporciona información sobre las actividades de promoción del mercado de trabajo, en relación a los diversos lugares y proyectos actuales. La estrategia futura del BFI consiste en satisfacer el mercado laboral europeo, que está en constante evolución y afronta nuevas y cambiantes necesidades con métodos didácticos basados en las TIC: la necesidad de movilidad, la flexibilidad, el aprendizaje en el lugar de trabajo, competencias y contenidos basados en el conocimiento, altos estándares de calidad y posibilidades para todos los grupos de edad de aprendizaje.

2 http://www.bfi.at/home/ 
No obstante, en el proceso de evolución del BFI se encontró que es muy difícil para los proveedores de vocational education and training (VET), que se configura como un sistema de educación dual inicial, proyectado más allá de localizar contenidos utilizables de alta calidad en otros idiomas -aparte del inglés- y de producir contenidos propios, el hecho de encontrar entrenadores con experiencia y con conocimientos técnicos de sonido en el campo del e-learning y del blended learning.

Asimismo, se configuraba como una tarea ardua el hecho de conseguir determinados formadores capacitados para producir contenido de aprendizaje y de introducir determinadas normas de calidad que tuvieran cumplimiento en todos los entrenamientos en línea. También era dificil encontrar contenidos abiertos de calidad elevada que puediesen ser utilizados y adaptados para dar cuenta de las necesidades regionales específicas. En resumen, la situación hasta ahora descrita era el escenario de dificultad en el que se movían los socios internacionales del BFI. Estos socios experimentaban estos problemas en todos los países e instituciones.

La Comisión Europea propuso acciones e innovaciones en la enseñanza y el aprendizaje mediante nuevas tecnologías y recursos educativos abiertos [Opening up education: innovative teaching and learning for all through new technologies and open educational resources (OER)]. Con esta medida, se ayuda al aprendizaje en las instituciones, a los profesores y a los estudiantes, de forma que adquieran competencias digitales y métodos de aprendizaje y apoyen el desarrollo y la disponibilidad de los recursos educativos abiertos. El apoyo a la producción de los OER es también una de las prioridades de la convocatoria Erasmus+.

Otra cuestión que se observó es la falta de contenido de aprendizaje basado en las TIC en el ámbito del «turismo sostenible», que se configura como una de las áreas con más crecimiento y potencial proyección en Europa. En esta área, los conocimientos del personal en su mayoría no cumplen con los requisitos establecidos, que era una de las principales conclusiones del BFI en un proyecto de cooperación territorial europea en curso sobre el turismo en Austria, que, como es bien sabido, se sitúa en la zona de la frontera checa. Este análisis también fue apoyado por

\section{(...) al igual que las TIC son habilidades esenciales para todo el mundo hoy en día, las competencias tecnológicas serán importantes para casi todos los trabajos en el futuro}

otros estudios. El Comunicado de Brujas sobre una cooperación europea reforzada en materia de educación y formación profesionales para el periodo 20112020 (efectuada por la reunión de ministros de Educación europeos y que tuvo lugar en Brujas en el año 2010) enfatiza con mucho interés la importancia de las habilidades relacionadas con la tecnología (Unión Europea, 2012). Este interés queda de manifiesto en las siguientes palabras: al igual que las TIC son habilidades esenciales para todo el mundo hoy en día, las competencias tecnológicas serán importantes para casi todos los trabajos en el futuro.

El proyecto combina la tecnología y las greenskills basadas en las TIC mediante la creación de los OER en el ámbito del turismo sostenible. Este enfoque se alinea con las estrategias nacionales de desarrollo económico en Austria. Dichas estrategias ven el turismo sostenible como un creciente sector empresarial (Oficina Austriaca de Turismo, 2012).

El escenario descrito tiene que ver con la necesidad educativa de la población de adquirir progresivamente habilidades en competencias digitales, todas ellas orientadas a la consecución de resultados. Por otra parte, cabe señalar que las competencias digitales (de las que podrían señalarse las habilidades digitales, la gestión de la información, el trabajo en red y el aprendizaje a lo largo de toda la vida, entre muchas otras) tienen como característica común el hecho de que se configuran como importantes herramientas tanto educativas como de negocio (Credé y Mansell, 1998; y Bisquerra, 2012). 


\section{LA INTERNACIONALIZACIÓN DE LA UDIMA, CONTEXTO PARA EL PROYECTO SOLA}

Tal y como se expresará de manera detallada en el siguiente epígrafe, destinado específicamente a la explicación del Proyecto SOLA, este proyecto se encuentra enmarcado dentro del denominado Programa Erasmus+ (KA2-Cooperation and Innovation for Good Practices), convocado por la Comisión Europea. Dentro de las nuevas necesidades de la docencia, el Proyecto SOLA tiene como objetivos prioritarios mejorar las competencias digitales de los docentes y la formación en las nuevas $\mathrm{TIC}^{3}$.

La internacionalización de la UDIMA se enmarca y adquiere su razón de ser dentro del contexto del Espacio Europeo de Educación Superior (EEES) y del escenario global de aperturismo de la educación universitaria a un entorno mundial, global, internacional (García, Mababu y Martínez, 2016). Así, la filosofía de nuestra universidad tiene como objetivo prioritario el logro de la excelencia académica, por lo que dotar a nuestros estudios del tinte internacional (y también a nuestra propia universidad) se configura como un reto y como una ambición, puesto que se ha entendido que la sociedad de la información 0 , más en concreto, la sociedad del conocimiento, tiene como factor ineludible la dimensión internacional, sobre todo por las importantes perspectivas laborales que ello proporciona. Hoy en día es importante tener un perfil internacional para conseguir una buena posición en el mercado de trabajo y del empleo. Ello es, en parte, porque vivimos en un mundo globalizado y la universidad se mueve en ese entorno novedoso.

La internacionalización, por tanto, es un factor necesario en el devenir de nuestra universidad. Por tanto, como institución joven y emergente, tiene su plan de internacionalización en pleno desarrollo, logrando cada vez un mayor auge. En este escenario, el Proyecto SOLA cuenta con un protagonismo ineludible, puesto que permite incrementar la colaboración con investigadores y docentes de otros países del mundo.

\footnotetext{
${ }^{3}$ http://www.udima.es/sites/udima.es/files/SOLA\%20project $\% 20 \% 28$ summary\%29_0.pdf
}

Tenemos que pensar que nos movemos en un escenario con unas coordenadas sociológicas que es preciso tener en cuenta. La realidad actual debe desarrollarse en la estrategia nacional de modernización de las universidades que ha recibido el nombre de Estrategia Universidad 2015 (EU2015). Esta señala de modo decidido que las universidades deben estar académicamente preparadas e internacionalizadas, con el objetivo de encarar positivamente el futuro. Por esta razón, unas instituciones universitarias que aboguen por la colaboración interuniversitaria a escala mundial (como es el caso de la UDIMA) lograrán una agregación y una colaboración estratégica organizativa que les resultará muy rentable a la hora de ser valoradas por la sociedad.

La UDIMA, en ese sentido, ha participado en el Programa Erasmus+ para el periodo 2014-2020 de la Comisión Europea, que require, como se puede leer en su propia página web4:

«Centrarse en el aprendizaje formal e informal más allá de las fronteras de la Unión Europea con una clara vocación de internacionalización, abriéndose a terceros países con el objetivo de mejorar las capacidades educativas y formativas de las personas para la empleabilidad de estudiantes, profesorado y trabajadores y trabajadoras».

\footnotetext{
4 http://www.udima.es/es/internacional.html
} 
Pasamos a continuación a explicitar de manera resumida los tres ejes de actuación más importantes que configuran el marco estratégico de la política de internacionalización de la UDIMA (dicha estrategia de internacionalización se circunscribe dentro del ámbito de la Estrategia Europa 2020 de la Unión Europea):

- Internacionalización de la cultura institucional.

- Docencia e investigación con dimensión internacional.

- Desarrollo de la movilidad y participación en las redes académicas internacionales.

Dentro del primer eje («internacionalización de la cultura institucional»), tenemos que el fomento de la competencia lingüística e intercultural de la comunidad universitaria es uno de los principales factores sobre los que pivota. Para ello, la colaboración con el Instituto de Idiomas de la universidad es crucial. También el apoyo a los miembros de nuestra comunidad para que realicen estancias en el extranjero es algo muy importante en esta estrategia.

En lo referente al segundo eje (recordemos que es «docencia e investigación con dimensión internacional»), nos encontramos con la promoción y el fomento de la participación en programas y en proyectos de $1+D+i$ internacionales de carácter interuniversitario y también con la promoción de actividades en colaboración con otras universidades y centros universitarios.

Por último, dentro del último eje (recordemos que es «desarrollo de la movilidad y participación en las redes académicas internacionales»), nos encontramos con que la UDIMA potencia la movilidad de nuestros estudiantes y también de nuestros profesores y de nuestro personal de administración y servicios, así como también fomenta su presencia, cada vez más activa, en redes universitarias de toda índole, siempre con esa dimensión global e internacional.

Para entender la dimensión internacional de la UDIMA es importante tener en cuenta el marco educativo global en el que nos estamos moviendo (al que ya hicimos alusión hace algunas líneas). Pensemos que nuestra estrategia de internacionalización está estructurada dentro de la Estrategia Europea 2020 de la Unión Europea. En este amplio escenario, es preciso tener en cuenta:

- La Estrategia de Movilidad 2020 para el EEES: movilidad para favorecer el aprendizaje activo, desa-

\section{(...) unas instituciones universitarias que aboguen por la colaboración interuniversitaria a escala mundial (como es el caso de la UDIMA) lograrán una agregación y una colaboración estratégica organizativa que les resultará muy rentable a la hora de ser valoradas por la sociedad}

rrollada y promovida a su vez por voluntad de los diferentes ministros de Educación de los países que conforman el EEES.

- La Estrategia de Cooperación Internacional en Educación Superior de la Unión Europea.

\section{EL PROYECTO SOLA}

Como ya hemos comentado anteriormente, el Proyecto SOLA es un proyecto aceptado dentro del programa Erasmus+ (KA2-Cooperation and Innovation for Good Practices), convocado por la Comisión Europea. Dentro de las necesidades de la docencia, el Proyecto SOLA tiene como objetivos prioritarios mejorar las competencias digitales de los docentes y la formación en las nuevas TIC, ayudar en la disposición y en el desarrollo de recursos abiertos educativos, vincular aulas entre sí e implementar dispositivos y contenidos digitales en las mismas, así como fomentar el uso de las TIC en instituciones educativas mediante movilización de profesores, estudiantes, socios económicos y familia ${ }^{5}$.

El proyecto SOLA propone alcanzar los siguientes objetivos principales:

- Formar «monitores de clase» que trabajen con las TIC y la pedagogía relacionada, que, junto con los métodos didácticos en varios contextos, consigan desarrollar y adaptar los contenidos concretos de e-learning.

\footnotetext{
${ }^{5}$ http://www.udima.es/sites/udima.es/files/SOLA\%20project\%20\%28summary\%29_0.pdf
} 
- Establecer normas de alta calidad para contenidos e-learning, seminarios y aulas fusionadas en conceptos de aprendizaje combinado.

- Desarrollar contenidos e-learning abiertos. Estos contenidos seguirán las normas específicas acordadas por todos los socios del proyecto.

- Desarrollar guías y manuales para utilizarlos en entornos de aprendizaje combinado.

- Aumentar la movilidad y el intercambio internacional de monitores, así como la comunicación de las organizaciones, la cooperación y el intercambio de experiencia y know-how.

- Ofrecer a los estudiantes la oportunidad de aprender a su propio ritmo con los métodos de aprendizaje basados en las TIC y con contenidos que están diseñados para un área específica en el idioma necesario.

- Desarrollar e implementar planes de estudios de alta calidad y módulos de aprendizaje en turismo sostenible, que se publican como OER.

- Introducir insignias abiertas y european credit transfer and accumulation system (ECTS) para reconocer los logros de aprendizaje.

- Lograr la participación de la industria del turismo y de las principales partes interesadas (como, por ejemplo, el servicio de empleo o los interlocutores sociales) en el proyecto.

De esta manera, el proyecto abarca principalmente los siguientes grupos destinatarios: los profesores y formadores en formación profesional (VET), los profesionales en turismo, los proveedores de formación profesional, la industria del turismo y sus representantes, y las partes interesadas (servicios de empleo, interlocutores sociales, etc.).

Con este proyecto se pretende
perfeccionar las competencias
digitales y las TIC del profesorado
participante, formar parte en la
creación y en el ofrecimiento de
contenidos y recursos didácticos,
así como en la movilización de
socios económicos y sociales

\section{EL CASO DE LA UDIMA EN EL PROYECTO SOLA}

La UDIMA participa en el Proyecto SOLA junto a otras instituciones formativas europeas de Alemania, Austria, Italia, Portugal, Países Bajos, Polonia y República Checa. Juntas han diseñado la estructura y los contenidos de varios cursos formativos en la modalidad semipresencial o blended learning, basados principalmente en las principales dimensiones de sostenibilidad del desarrollo turístico, medioambiental, económico y sociocultural ${ }^{6}$.

Con este proyecto, se pretende perfeccionar las competencias digitales y las TIC del profesorado participante, formar parte en la creación y en el ofrecimiento de contenidos y recursos didácticos, así como en la movilización de socios económicos y sociales con el propósito de promover el uso de las TIC para la formación del profesorado. Además, este proyecto tiene como objetivo crear contenidos pedagógicos TIC, sobre todo en el área del turismo sostenible, el cual, actualmente, es un campo de gran crecimiento en Europa. Los cursos on-line diseñados para este proyecto son los siguientes:

- Conceptos básicos del turismo sostenible.

- Gestión de las relaciones con el cliente en turismo sostenible.

- Turismo sostenible rural.

En la UDIMA, el Proyecto SOLA cuenta con la participación de Raquel García Revilla y de Gerardo Ravassa Escobar y se seguirá llevando a cabo durante los próximos años. Asimismo, los socios de la UDIMA en este proyecto europeo son los siguientes:

- Berufsförderungsinstitut Oberoesterreich, en Austria $(\mathrm{BFI})^{7}$.

- Akademia Humanistyczno-Ekonomiczna w Lodzi, en Polonia (AHE) $)^{8}$.

- Istituto Formazione Operatori Aziendali, en Italia $(\text { IFOA })^{9}$.

\footnotetext{
${ }^{6}$ http://www.udima.es/sites/udima.es/files/SOLA $\% 20$ project $\% 20 \% 28$ summary\%29_0.pdf

${ }^{7}$ http://www.bfi.at/home/

${ }^{8} \mathrm{http}: / / \mathrm{www} \cdot$ ahe.lodz.pl/

9 http://www.ifoa.it/
} 


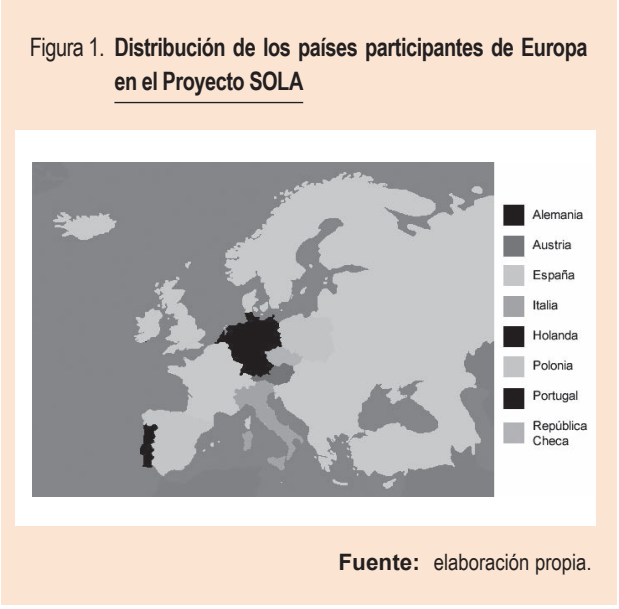

- Instituto de Soldadura e Qualidade, en Portugal $(\mathrm{ISQ})^{10}$.

- Link \& Link Software, en Alemania (LL) ${ }^{11}$.

- Stichting ROC Midden Nederland, en los Países Bajos $(R O C)^{12}$.

- Tempo Training \& Consulting, en la República Checa $(\text { TTT })^{13}$.

En la figura 1 se presenta la distribución de los países de Europa que participan en el proyecto SOLA.

\footnotetext{
10 http://www.isq.pt/

11 http://www.linkundlink.de/

12 http://www.rocmn.nl

13 http://www.tempo.cz/
}

\section{BIBLIOGRAFÍA}

Bisquerra, R. (coord.) [2012]: Metodología de la investigación educativa, Madrid: La Muralla.

Credé, A. y Mansell, R. [1998]: Knowledges societies: information technologies for susteinable development, Otawa: International Development Center.

Crovi, D. [mayo-agosto 2002]: «Sociedad de la información y el conocimiento. Entre el optimismo y la desesperanza», Revista Mexicana de Ciencias Sociales y Políticas, XLV (198), págs. 13-34.

\section{CONCLUSIONES}

La UDIMA participa en el proyecto SOLA junto a otras instituciones formativas europeas, y todas ellas han diseñado la estructura y los contenidos de varios cursos formativos en la modalidad semipresencial o blended learning, establecidos mayoritariamente sobre las principales dimensiones de sostenibilidad del desarrollo turístico, medioambiental, económico y sociocultural.

Este proyecto trae consigo interesantes aportaciones, en especial para la UDIMA. Entre ellas destacan que el blended learning dota de habilidades al profesorado y permite una docencia de calidad sin barreras, a la vez que perfecciona las competencias digitales y las TIC del mismo utilizando contenido audiovisual. En general, mediante un proceso de aprendizaje con un aula virtual se obtiene una organización del espacio y del tiempo educativo menos definida, se hace un uso sistemático e intenso de las nuevas TIC, los contenidos didácticos se pueden apoyar en la tecnología y la interacción social se produce de una forma telemática.

Asimismo, este proyecto, debido a que hace que el aprendizaje sea colaborativo y dinámico entre los estudiantes, ayuda a los participantes a fomentar la atención a la diversidad a través de las actividades en el aula virtual, como, por ejemplo, a los estudiantes con necesidades especiales; colabora con la universalización de la institución universitaria; ayuda a que haya siempre una actualización constante y rigurosa de los contenidos, y favorece el tránsito entre la universidad y el mundo laboral.

Hasta el momento, se ha alcanzado el desarrollo y la calidad esperados gracias a las herramientas e instrumentos con los que cuenta la enseñanza virtual.

García, M. R.; Mababu, R. y Martínez, O. [2016]: The new reality of the spanish university: new spaces for new pedagogies, EDULEARN16. Pedagogical Innovations and International Projects.

Unión Europea [2012]: Comunicado de Brujas sobre una cooperación europea reforzada en materia de educación y formación profesionales para el periodo 20112020, págs. 8-19. 